\title{
Silanol Effect on the Chromatographic Behavior of Tris(2-methyl-8-quinolinolato)gallium in High-Performance Liquid Chromatography
}

\author{
Nobuo Uehara, Toshifumi Kurahashi and Yoshio ShIJo \\ Department of Applied Chemistry, Faculty of Engineering, Utsunomiya University, \\ Ishiicho, Utsunomiya 321, Japan
}

\begin{abstract}
The chromatographic behavior of tris(2-methyl-8-quinolinolato)gallium and other tris(methyl-substituted 8quinolinolato)galliums was studied with a methanol-water mixture $(80: 20 \mathrm{v} / \mathrm{v})$ containing $5 \times 10^{-4} \mathrm{~mol} \mathrm{dm}^{-3}$ of the corresponding methyl-substituted 8-quinolinol and $2 \times 10^{-3} \mathrm{~mol} \mathrm{dm}^{-3}$ of a hexamethylenetetramine buffer (pH 6.0). The chromatographic behavior of tris(2-methyl-8-quinolinolato)gallium and tris(2,5-dimethyl-8-quinolinolato)gallium changed when the column was varied. The other tris(methyl-substituted 8-quinolinolato)galliums did not change. Tris(2-methyl-8-quinolinolato)gallium and tris(2,5-dimethyl-8-quinolinolato)gallium were dissociated by EDTA among all of the tris(methyl-substituted 8-quinolinolato)galliums studied. It was suggested that the chromatographic behavior of tris(2-methyl-8-quinolinolato)gallium was influenced by the chelate forming the interaction of the silanol group on the stationary phase.
\end{abstract}

Keywords Tris(2-methyl-8-quinolinolato)gallium, silanol group, chelate-forming interaction, high-performance liquid chromatography

The separation and determination of metal chelates with high-performance liquid chromatography (HPLC) is one of the attractive methods for the determination of metal ions. ${ }^{1-4}$ A precolumn derivatization technique has been popularly used in separation studies of metal chelates with HPLC because of its high reproducibility and sensitivity. Generally, the chelating agents were added to the eluents in order to suppress any dissociation of the chelates for a simultaneous determination with the precolumn derivatization technique. However, every chelate injected into the HPLC column was not separated under the conditions in which the dissociation was suppressed. The chromatographic behavior of the noneluting chelate has not been given much attention.

The second effect of the silanol group on the stationary phase influenced the chromatographic behavior of the metal chelates. Although the ion-exchange interaction of the silanol group toward cation chelates has been extensively investigated ${ }^{5,6}$, studies concerning the chelate-forming interaction of the silanol group have been only qualitative. ${ }^{7,8}$ Suitable metal chelates for studying chelate-forming interactions have not yet been found. This paper describes the silanol effect on the chromatographic behavior of tris(2-methyl-8-quinolinolato)gallium and other tris(methyl-substituted 8quinolinolato)galliums.

\section{Experimental}

Apparatus

The HPLC system comprised of a Shimadzu LC-6A pump (Kyoto, Japan), a Japan Spectroscopic 870-UV spectrophotometer (Tokyo, Japan), a Shimadzu RF-535 fluorometric detector, a Rheodyne 7125 loop injector equipped with a $20 \mu \mathrm{l}$ sample loop, and a Shimadzu R1231 recorder.

Ten commercially available columns were used. A Cosmosil $5 \mathrm{C}_{18}$ column $(150 \mathrm{~mm} \times 4.6 \mathrm{~mm}$ i.d. $)$, a Cosmosil 5TMS column $(150 \mathrm{~mm} \times 4.6 \mathrm{~mm}$ i.d. $)$, and a Cosmosil 5SL column $(150 \mathrm{~mm} \times 4.6 \mathrm{~mm}$ i.d. $)$ were obtained from Nacarai Tesque (Kyoto, Japan). A TSKgel ODS 80TM column $(150 \mathrm{~mm} \times 4.6 \mathrm{~mm}$ i.d. $)$ and a TSKgel octadecyl-4PW column $(150 \mathrm{~mm} \times 4.6 \mathrm{~mm}$ i.d. $)$ were obtained from TOSOH (Tokyo, Japan). An Lcolumn ODS ( $150 \mathrm{~mm} \times 4.6 \mathrm{~mm}$ i.d.), a Partisil 5 ODS-3 column (150 mm $\times 4.6 \mathrm{~mm}$ i.d.), an LiChrospher $100 \mathrm{RP}$ 18(e) $(125 \mathrm{~mm} \times 4 \mathrm{~mm}$ i.d.), and a YMC R-ODS-5 ( $250 \mathrm{~mm} \times 4.6 \mathrm{~mm}$ i.d.) were obtained from Kagakuhin Kensa Kyoukai (Tokyo, Japan), Watman, Merck, and YMC (Kyoto, Japan), respectively. A Capcell SG 120 column was prepared by packing Capcell SG 120 (Shiseido, Tokyo, Japan) into a stainless-steel column (150 $\mathrm{mm} \times 4.6 \mathrm{~mm}$ i.d.). 


\section{Materials}

8-Quinolinol and 2-methyl-8-quinolinol were obtained from Kanto Chemicals (Tokyo, Japan) and recrystallized from ethanol. Other methyl-substituted 8-quinolinols were synthesized in the laboratory. All methylsubstituted 8-quinolinol was dissolved to methanol. A standard solution of gallium(III) $\left(0.01 \mathrm{~mol} \mathrm{dm}^{-3}\right)$ was prepared by dissolving gallium nitrate in $0.01 \mathrm{~mol} \mathrm{dm}^{-3}$ nitric acid and standardized by titration with ethylenediaminetetraacetic acid. Distilled, deionized water was further purified by a Millipore Milli-Q system. Analytical-reagent grade methanol was passed through a Millipore filter (pore size $0.45 \mu \mathrm{m}$ ) after distillation. Cosmosil 5TMS and 5SL packing materials were obtained from Nacarai Tesque (Kyoto, Japan). All other reagents used were of analytical reagent grade.

\section{Procedure}

To $1 \mathrm{ml}$ of a $1 \times 10^{-4} \mathrm{~mol} \mathrm{dm}^{-3}$ gallium(III) solution in a $20 \mathrm{ml}$ volumetric flask, $1 \mathrm{ml}$ of $5 \times 10^{-2} \mathrm{~mol} \mathrm{dm}^{-3} 2$ methyl-8-quinolinol solution, $2 \mathrm{ml}$ of $1.0 \mathrm{~mol} \mathrm{dm}^{-3}$ tris(hydroxymethyl)aminomethane buffer ( $\mathrm{pH} \mathrm{8.0)}$, and $10 \mathrm{ml}$ of methanol were added. After the solution had been diluted to the mark with water, an aliquot $(20 \mu \mathrm{l})$ of the solution was injected into the HPLC system.

The eluent was methanol-water $(80: 20 \mathrm{v} / \mathrm{v})$ containing $5 \times 10^{-4} \mathrm{~mol} \mathrm{dm}^{-3}$ methyl-substituted 8-quinolinol and $2 \times 10^{-3} \mathrm{~mol} \mathrm{dm}^{-3}$ hexamethylenetetramine. The $\mathrm{pH}$ of the eluent was adjusted to 6.0 by the addition of hydrochloric acid prior to the addition of methanol. The flow rate was $0.8 \mathrm{ml} \mathrm{min}-1$. The excitation and emission wavelengths were set at 365 and $510 \mathrm{~nm}$, respectively.

\section{Results and Discussion}

\section{Chelate formation and chromatographic conditions}

All of the methyl-substituted 8-quinolinols studied formed non-charged chelates with gallium ions in the neutral region. A methanol-water $(50: 50 \mathrm{v} / \mathrm{v})$ mixture was used to dissolve the Ga-chelates. Since all of the Ga-chelates were fluorescent, fluorescent detection was used. Fluorescent detection was advantageous for monitoring Ga-chelates because the background due to methyl-substituted 8-quinolinol was very low and high sensitivity was achieved, which permitted identification of the broad peak of the Ga-chelate from the base line. Since the fluorescence of tris(5,7-dimethyl-8-quinolinolato)gallium was very weak, the chelate was monitored by spectrophotometric detection.

Figure 1 shows a typical chromatogram of tris(2methyl-8-quinolinolato)gallium (Ga-2mQ) using Cosmosil $5 \mathrm{C}_{18}$. The methyl-substituted 8-quinolinols were added to the eluents in order to suppress any dissociation of the Ga-chelates. All of the Ga-chelates were stable in the eluent, so that the effect of dissociation could be omitted when considering the chromatographic behavior of the Ga-chelates. The retention time of

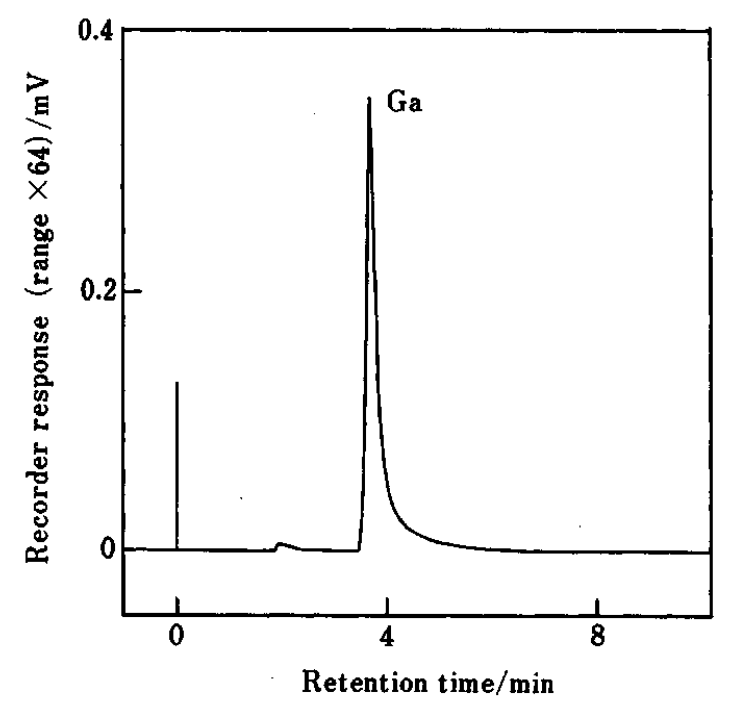

Fig. 1 Typical chromatogram of tris(2-methyl-8-quinolinolato)gallium. Column, Cosmosil $5 \mathrm{C}_{18}(150 \mathrm{~mm} \times 4.6 \mathrm{~mm}$ i.d. $)$; eluent, methanol-water $(80: 20 \mathrm{v} / \mathrm{v})$ containing $5 \times 10^{-4}$ mol dm${ }^{-3}$ 2-methyl-8-quinolinol and $2 \times 10^{-3} \mathrm{~mol} \mathrm{dm}^{-3}$ hexamethylenetetramine ( $\mathrm{pH} 6.0$ ); $\mathrm{Ga}, 5 \times 10^{-6} \mathrm{~mol} \mathrm{dm}^{-3}$; injection volume $20 \mu \mathrm{l}$; detection wavelengths, Ex $365 \mathrm{~nm}$, Em $510 \mathrm{~nm}$; flow rate, $0.8 \mathrm{ml} \mathrm{min}^{-1}$.

every Ga-chelate decreased with increasing methanol content in the eluent. All of the Ga-chelates were eluted within $7 \mathrm{~min}$ with all of the columns used.

\section{Influence of the packing materials}

Table 1 shows the chromatographic behavior of Ga$2 \mathrm{mQ}$ in terms of the peak shapes with various commercially available packed columns. The Cosmosil 5SL column was a column packed with silica gel. The Cosmosil 5TMS column was a column packed with trimethylsilyl silica gel. The TSKgel octadecyl-4PW was a polymer-gel column. The Capcell SG 120 was a polymer-coated silica gel having a $\mathrm{C}_{18}$ group. The other columns were ordinary ODS columns with endcapping treatment. Cosmosil $5 \mathrm{C}_{18}$, Cosmosil 5TMS, and TSKgel Octadecyl 4PW gave a good peak of Ga$2 \mathrm{mQ}$ among all of the columns used here. Judging from

Table 1 Peak shapes of tris(2-methyl-8-quinolinolato)gallium with various columns

\begin{tabular}{cl}
\hline Peak shape & \multicolumn{1}{c}{ Column used } \\
\hline Good peak & Cosmosil 5C18 \\
& Cosmosil 5TMS \\
& TSKgel Octadecyl-4PW \\
Broaden peak & L-Column ODS \\
& Capcell SG-120 \\
No peak & LiChrospher RP-18(e) \\
& Partisil 5 ODS-3 \\
& TSKgel ODS-80T \\
& YMC R-ODS-5 \\
& Cosmosil 5SL \\
\hline
\end{tabular}

Chromatographic conditions are same as Fig. 1. 


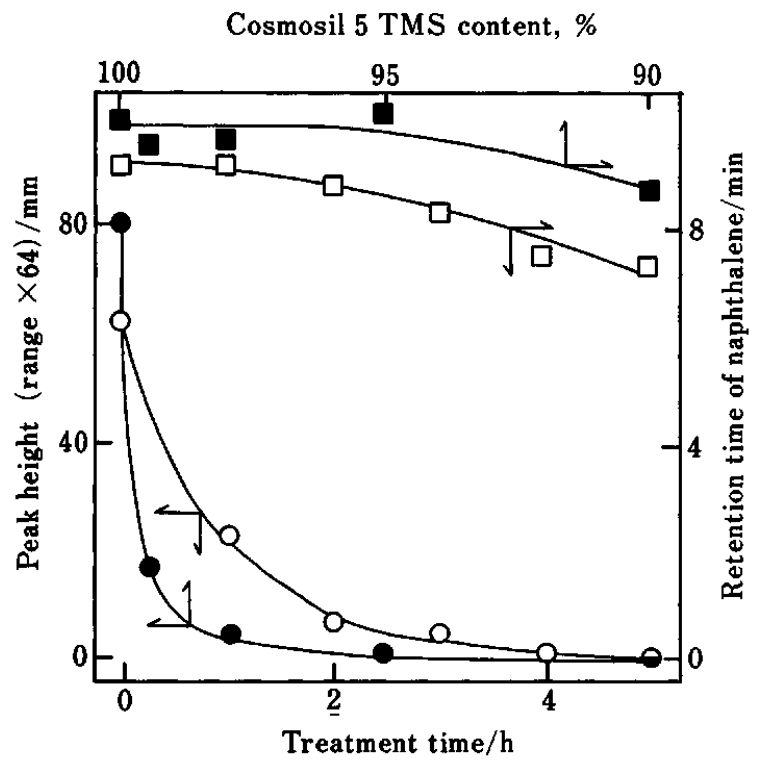

Fig. 2 Influence of the alkaline treatment and Cosmosil 5TMS content against the peak height of tris(2-methyl-8quinolinolato) gallium and the retention time of naphthalene. Column, Cosmosil 5TMS and Cosmosil 5TMS+Cosmosil 5SL (150 $\times 4.6 \mathrm{~mm}$ i.d.); eluent for naphthalene, methanol-

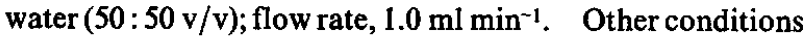
as in Fig. 1. Peak height of tris(2-methyl-8-quinolinolato)gallium with the "mixed material column" $(\bullet)$; peak height of tris(2-methyl-8-quinolinolato)gallium with the "alkali treatment column" $(O)$; retention time of naphtalene with the "mixed material column" ( $\square$ ); and retention time of naphtalene with the "alkali treatment column" $(\square)$.

the elution behavior with the L-column ODS and Capcell SG-120, which gave very broad peaks, the Ga-2mQ eluted very gradually with the other ODS columns. Therefore, the peak could not be identified from the base line.

Comparing the result from the Cosmosil 5TMS column with that from the Cosmosil 5SL column, it was presumed that the silanol group affected the chromatographic behavior of Ga-2mQ. Two types of columns having different amounts of the silanol group were examined in order to study the influence of the silanol group on the elution of Ga-2mQ. One type of column (called an "alkali treatment column") was prepared by treatment of the Cosmosil 5TMS column $(150 \mathrm{~mm} \times$ $4.6 \mathrm{~mm}$ i.d.) with an alkali solution. The alkali solution (methanol-water 80:20 v/v, pH 9.0) was passed through the Cosmosil 5TMS column for different lengths of time. The other type of column (called a "mixed material column") was prepared with the packing material mixed with Cosmosil 5TMS and Cosmosil 5SL. The results obtained with both types of columns are given in Fig. 2 . Figure 2 also includes the change in the retention time of naphthalene with both types of columns. The decrease in the retention time of naphthalene corresponded to the decrease in the amount of the trimethylsilyl groups. Hydrolysis of the trimethylsilyl group produced the silanol group. The silanol group on the "mixed material

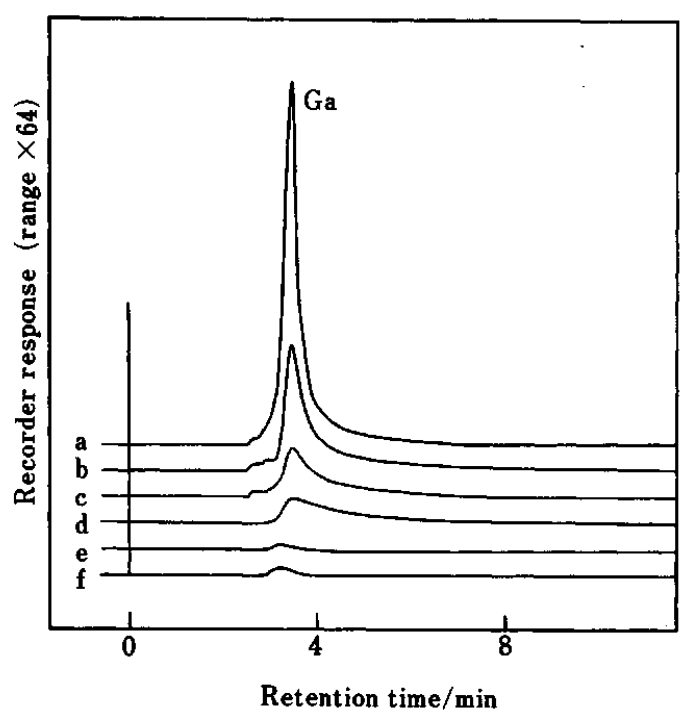

Fig. 3 Change of chromatograms of tris(2-methyl-8quinolinolato)gallium with alkaline treatment of column. Column, Cosmosil 5TMS (150 mm $\times 4.6 \mathrm{~mm}$ i.d.); other chromatographic conditions as in Fig. 1. Alkaline solutions, methanol-water $(80: 20 \mathrm{v} / \mathrm{v})$ containing $2 \times 10^{-3} \mathrm{~mol}$ $\mathrm{dm}^{-3}$ ammonium acetate (pH 9.0). Treatment time: a, 0 h; b, 1 h; c, 2 h; d, 3 h; e, 4 h; f, 5 h.

Table 2 Chromatographic behavior of methyl-substituted 8-quinolinol-gallium(III) chelates

\begin{tabular}{lccc}
\hline $\begin{array}{c}\text { Methyl-substituted } \\
\text { 8-quinolinol }\end{array}$ & $\begin{array}{c}\text { LiChrospher } \\
\text { RP-18(e) }\end{array}$ & $\begin{array}{c}\text { L-Column } \\
\text { ODS }\end{array}$ & $\begin{array}{c}\text { Cosmosil } \\
\text { 5C }_{\mathbf{1 8}}\end{array}$ \\
\hline 8-quinolinol & $\bigcirc$ & $\bigcirc$ & $\bigcirc$ \\
2-methyl- & $\times$ & $\triangle$ & $\bigcirc$ \\
3-methyl- & $\bigcirc$ & $\bigcirc$ & $\bigcirc$ \\
4-methyl- & $\bigcirc$ & $\bigcirc$ & $\bigcirc$ \\
5-methyl- & $\bigcirc$ & $\bigcirc$ & $\bigcirc$ \\
6-methyl- & $\bigcirc$ & $\bigcirc$ & $\bigcirc$ \\
2,5-dimethyl- & $\times$ & $\times$ & $\bigcirc$ \\
5,7-dimethyl-a & $\bigcirc$ & $\bigcirc$ & $\bigcirc$ \\
\hline
\end{tabular}

$\bigcirc$, good peak; $\Delta$, broad peak with tailing; $X$, no peak. a. spectrophotometric detection. Chromatographic conditions except 8-quinolinols are same as Fig. 1.

column" influenced the chromatographic behavior of Ga-2mQ more effectively than did the silanol group on the "alkali treatment column". Figure 3 shows the visual change in the chromatograms of $\mathrm{Ga}-2 \mathrm{mQ}$ using the "alkali treatment column".

\section{Chromatographic behavior of other Ga-chelates}

The chromatographic behavior of other Ga-chelates was investigated using three ODS columns. The results are summarized in Table 2. Tris(2,5-dimethyl-8quinolinolato)gallium $(\mathrm{Ga}-2,5 \mathrm{mQ})$ gave the same result as did $\mathrm{Ga}-2 \mathrm{mQ}$. The other Ga-chelates exhibited good peaks with all of the ODS columns used here. It was reported $^{9}$ that the 2-methyl group introduced into 8quinolinol made their chelates unstable. The thermo- 


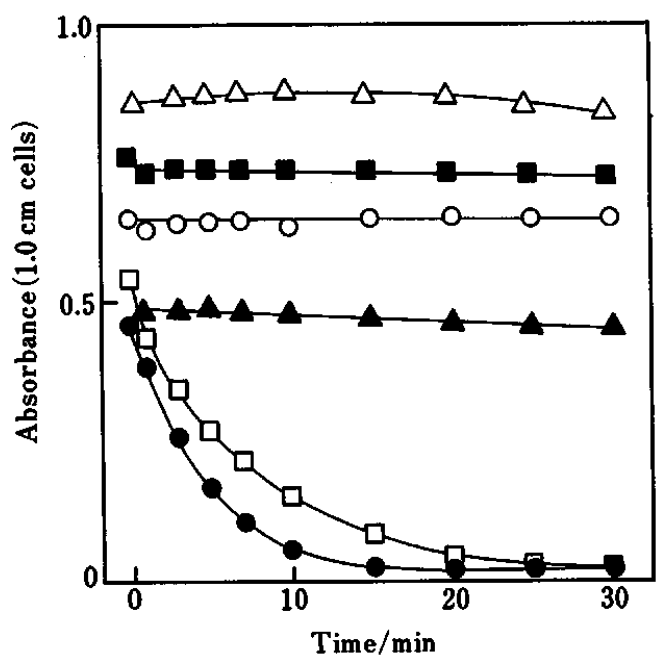

Fig. 4 Absorbance change of Ga-chelates with EDTA. Ga, $1 \times 10^{-4} \mathrm{~mol} \mathrm{dm}^{-3}$; methyl-substituted 8-quinolinols, $1 \times 10^{-3}$ mol dm$m^{-3}$; EDTA, $1 \times 10^{-3} \mathrm{~mol} \mathrm{dm}^{-3}$; tris(hydroxymethyl)aminomethane, $0.05 \mathrm{~mol} \mathrm{dm}^{-3}$; methanol-water $(50: 50 \mathrm{v} / \mathrm{v})$; pH, 8.0. O, 8-quinolinol; 9 , 2-methyl-8-quinolinol; $\mathbf{\square}$, 3methyl-8-quinolinol; $\Delta, 4$-methyl-8-quinolinol; $\Delta, 6$-methyl8-quinolinol; $\square$, 2,5-dimethyl-8-quinolinol.

dynamic stability of the chelates seemed to affect the elution of $\mathrm{Ga}-2 \mathrm{mQ}$.

Tetrabutylammonium bromide ${ }^{6}$ and aluminum chloride were used to study the ion-exchange interaction and the chelate-forming interaction of the silanol group, respectively. However, the blocking effects of both tetrabutylammonium bromide and aluminum chloride were not observed.

The retention behavior of other 2-methyl-8-quinolinol (2mHQ) chelates of $\mathrm{Cr}^{\mathrm{III}}, \mathrm{V}^{\mathrm{v}}, \mathrm{Zn}^{\mathrm{II}}, \mathrm{Co}^{\mathrm{II}}, \mathrm{Cu}^{\mathrm{II}}, \mathrm{Ni}^{\mathrm{III}}, \mathrm{Fe}^{\mathrm{III}}$ and $\mathrm{In}^{\mathrm{III}}$ was investigated by HPLC with spectrophotometric detection. Results similar to those for Ga-2mQ were obtained with $\mathrm{V}^{\mathrm{V}}$ and $\mathrm{Fe}^{\mathrm{III}}$, which were "hard acid" and oxophilic metal ions. $\mathrm{Ga}^{\mathrm{III}}, \mathrm{V}^{\mathrm{V}}$ and $\mathrm{Fe}^{\mathrm{III}}$ seemed to prefer the silanol group to $2 \mathrm{mHQ}$.

\section{Influence of EDTA and silica gel}

The influence of an ethylenediaminetetraacetic acid (EDTA) and a silica gel on the Ga-chelates was studied spectrophotometrically. Figure 4 shows the ligand exchange reaction of the Ga-chelates with EDTA. Only $\mathrm{Ga}-2 \mathrm{mQ}$ and $\mathrm{Ga}-2,5 \mathrm{mQ}$ chelates were rapidly dissociated by EDTA. These results are consistent with those given in Table 2. The 2-methyl group in 8-quinolinol caused the Ga-chelates not only to be thermodynamically unstable, but also to be kinetically labile.

Silica gel was added to an aqueous-methanol mixture containing $1.0 \times 10^{-4} \mathrm{~mol} \mathrm{dm}^{-3}$ gallium(III) nitrate and $1.0 \times 10^{-4} \mathrm{~mol} \mathrm{dm}^{-3} 2 \mathrm{mHQ}$. Figure 5 shows the change in the absorption spectra of $\mathrm{Ga}-2 \mathrm{mQ}$. As the amount of silica gel added was increased, the spectra shifted from $\mathrm{Ga}-2 \mathrm{mQ}$ to $2 \mathrm{mHQ}$. The isobestic point was not observed because of the background of silica-gel scattering. The release of $2 \mathrm{mHQ}$ from $\mathrm{Ga}-2 \mathrm{mQ}$ upon

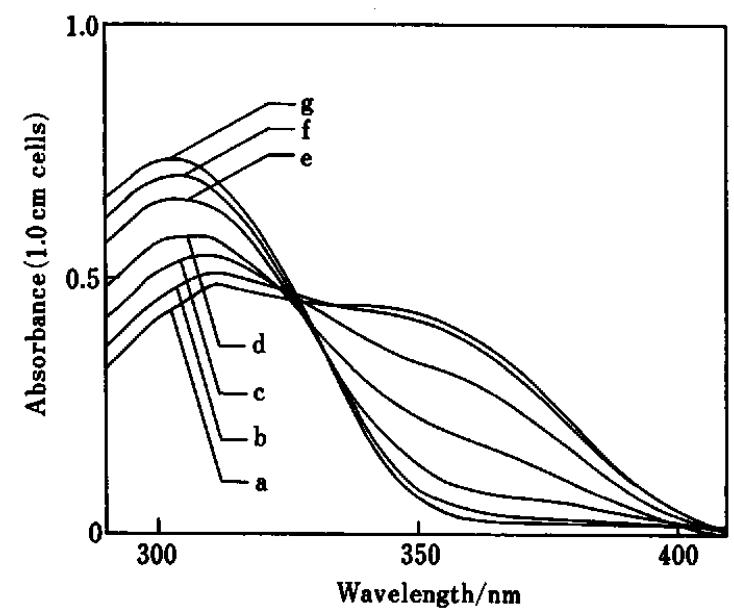

Fig. 5 Spectra change of tris(2-methyl-8-quinolinolato)gallium with silica gel. Ga, $1 \times 10^{-4} \mathrm{~mol} \mathrm{dm}^{-3}$; 2-methyl-8quinolinol, $1 \times 10^{-4} \mathrm{~mol} \mathrm{dm}^{-3}$; tris(hydroxymethyl)aminomethane, $0.1 \mathrm{~mol} \mathrm{dm}^{-3}$; methanol-water $(50: 50 \mathrm{v} / \mathrm{v}) ; \mathrm{pH}$, 8.0. Silica gel (100-200 mesh); a, $0 \mathrm{~g} ; \mathrm{b}, 0.25 \mathrm{~g} ; \mathrm{c}, 0.5 \mathrm{~g}$; $\mathrm{d}, 0.75 \mathrm{~g} ; \mathrm{e}, 1.0 \mathrm{~g} ; \mathrm{f}, 1.25 \mathrm{~g} ; \mathrm{g}, 1.5 \mathrm{~g}$.

adding silica gel suggested the existence of a ligand exchange reaction of $\mathrm{Ga}-2 \mathrm{mQ}$ with the silanol groups. No change in the spectra of tris(8-quinolinolato)gallium (Ga-Q) upon adding silica gel was observed.

The chromatographic behavior of $\mathrm{Ga}-2 \mathrm{mQ}$ and other Ga-chelates was influenced by the silanol group. A chelate-forming interaction of the silanol group was suggested in this work. The chelates may become possible probes for detecting the silanol group in HPLC columns. That will be a subject of further work.

The authors are grateful to the Ministry of Education, Science, and Culture of Japan for financial support (Grant No. 03855151).

\section{References}

1. B. R. Willeford and H. Veening, J. Chromatogr., 251, 61 (1982).

2. J. W. O'Laughlin, J. Liq. Chromatogr., 7, 127 (1984).

3. G. Nickless, J. Chromatogr., 313, 129 (1985).

4. K. Robards, P. Starr and E. Patsalides, Analyst [London], 116, 1247 (1991).

5. N. Suzuki, T. Takeda and K. Saitoh, Chromatographia, 22, 43 (1986).

6. J. Miura, Anal. Chem., 62, 1424 (1990).

7. M. D. Palmieri and J. S. Fritz, Anal. Chem., 59, 2226, (1987).

8. M. D. Palmieri and J. S. Fritz, Anal. Chem., 60, 2244 (1988).

9. T. Yotsuyanagi, Y. Katsura and T. Kudo, Abstract of papers, International Congress on Analytical Sciences 1991, Chiba, August 1991, p. 51.

(Received June 17, 1993)

(Accepted November 30, 1993) 